\title{
Catalpol Upregulates Hippocampal GAP-43 Level of Aged Rats with Enhanced Spatial Memory and Behavior Response
}

\author{
Jing Liu ${ }^{1 *}$, Yang Liu ${ }^{1}$, Wei Zou ${ }^{2}$, Lin Song ${ }^{1,3}$, Lijia $\mathrm{An}^{3}$ \\ ${ }^{1}$ Sino-UK Regenerative Medicine Center, The First Affiliated Hospital of Dalian Medical University, Dalian, China \\ ${ }^{2}$ Department of Biology, Liaoning Normal University, Dalian, China \\ ${ }^{3}$ School of Environmental and Biological Science, Dalian University of Technology, Dalian, China \\ Email: "dngs008@yahoo.com.cn
}

Received August 5, 2012; revised September 7, 2012; accepted September 17, 2012

\begin{abstract}
Rehmannia glutinosa is a traditional Chinese medical herb and has a long history in cognitive deficits therapy. Its therapeutic efficacy has been confirmed by clinical studies. In this study, we attempted to investigate the effects of catalpol, an iridoid from Rehmannia glutinosa, on cognitive and behavioral function of aged rats with memory loss. 22 24 month Sprague-Dawley spontaneous rats of memory loss with aging were selected by step-down type passive avoidance test and randomly allocated to two groups: the aged rats with memory loss (control group) and the catalpol-treated $(5 \mathrm{mg} / \mathrm{kg})$ group. We performed open-field and Y-maze test to evaluate special performance and behavior response before and after catalpol treatment for 5 and 10 days. Growth-associated protein (GAP-43) in hippocampus and frontal cortex was measured using immunohistochemistry and quantitative Western Blotting. The results showed that catalpol could significantly improve not only spatial learning and memory but also locomotor activity and exploratory behavior of aged rats with memory loss. GAP-43 protein in hippocampal CA3 region and dentate granule of catalpol-treated rats was significantly enhanced than that of control group. Western blot analysis demonstrated a catalpol-associated increase of GAP-43 in hippocampus of catalpol-treated rats and correlated with spatial memory, locomotor activity and exploratory behavior. However, there was no difference in GAP-43 protein in frontal cortex between two groups. These results indicated that catalpol could enhance spatial performance and behavioral responses in aged rats with memory loss, and the mechanism may involve up-regulation of GAP-43 level of hippocampus in the brain. It also suggested that catalpol may be a useful natural drug for Alzheimer's disease (AD) treatment by modulating hippocampal neuroplasticity.
\end{abstract}

Keywords: Catalpol; GAP-43 Protein; Neuroplasticity; Behavior; Memory Loss with Aging

\section{Introduction}

Declines in cognitive function such as memory loss are common with aging. The age-related memory loss is a normal process of aging but also may be the result of neurodegenerative disorders such as Alzheimer's disease (AD). As the life span is being prolonged with the advance in medical science, the incidence of aged-related memory loss has been dramatically risen.

The cause of aged-related memory loss has been thought a degeneration of brain function of the patients. New treatment strategies of aged-related memory loss based upon a better understanding of the inherent mechanisms of neuroplasticity might provide more rational approaches to deal with it and other neurodegenerative disorders. Neuroplasticity is both a substrate of learning and mem-

${ }^{*}$ Corresponding author. ory and a mediator of responses to neuronal attrition and injury (compensatory plasticity). Recent experiments by researchers have demonstrated that GAP-43, a molecular marker of central neuroplasticity [1], decreased in different brain regions in age-related memory loss patients. Although the relationship between molecular neuropathologic changes and cognitive disturbances remains obscure, there is extensive evidence suggesting that dysfunction of the central neuroplasiticity contributes to the cognitive impairment [2]. Rekart et al. observed that reducing hippocampus GAP-43 level contributes to memory impairment in aged-related memory loss [3]. The rapid progress towards understanding the cellular and molecular alterations in neuroplasticity that is responseble for the neuron's demise may help in developing effective preventative and therapeutic strategies, which may modify the traditional pharmaceutical drug devel- 
opment for aged-related memory loss. To date, there is no convincing evidence of efficacy for any drug.

Rehmannia glutinosa is a traditional Chinese medical herb and has a long history in cognitive defect therapy and its therapeutic efficacy has been confirmed by clinical studies. Catalpol (Figure 1) is a major active ingredient contained richly in the roots of rehmannia glutinosa. The previous work has indicated catalpol is able to increase learning ability of global ischemia model by performing neuroprotective effects [4], and protect PC12 cells from damage induced by $\mathrm{H}_{2} \mathrm{O}_{2}$ [5]. Therefore, we think that catalpol might improve cognition deficits in aged rats with memory loss by performing protective effects.

Since it is well accepted that cognitive impairment observed in aged-related memory loss is associated with decreasing central neuroplasticity, we have reason to assume that catalpol may have effect on neuroplasticity in aged brain if it can increase cognitive function, whereas studies elaborating the role of catalpol in neuroplasticity are completely lacking. In this study, we aim to examine the influence of catalpol on spontaneous aged rats with memory loss on both spatial memory and behavioral responses, which may throw some light on exploring a possible mechanism of catapol action and a promising treatment for $\mathrm{AD}$.

\section{Materials and Methods}

\subsection{Animals and Diets}

22 - 24 month male rats (Sprague-Dawley, 400 - $650 \mathrm{~g}$ ) and 4-month young male rats (Sprague-Dawley, 300 $350 \mathrm{~g}$ ) were obtained from the breeding colony of the Dalian Medical University, China. Four rats were housed per cage, and in a controlled environment with a temperature at $21^{\circ} \mathrm{C} \pm 1{ }^{\circ} \mathrm{C}$ and a $12 / 12 \mathrm{~h}$ dark-light cycle with light was on at $7 \mathrm{am}$. The animals had free access to food and water.

\subsection{Selected Aged Rats with Memory Loss}

Step-down type passive avoidance test: The apparatus

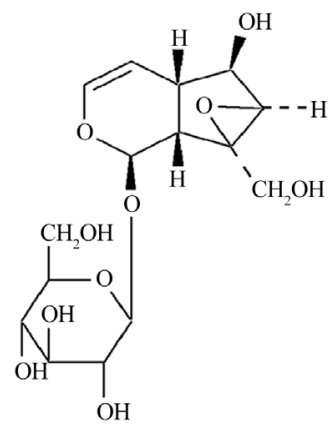

Figure 1. The chemical structure of catalpol. consisted of acrylic box with a stainless-steel grid floor. A platform was fixed in the end of the box. Electric shocks $(36 \mathrm{~V})$ were delivered to the grid floor with an isolated pulse stimulator. At the beginning of training trial, rats were placed in the box to adapt for $3 \mathrm{~min}$. When electric shocks were delivered, rats jumped to platform. The shocks were maintained for $5 \mathrm{~min}$. Step-down latency (SDL) was recorded within $5 \mathrm{~min}$. A 99\% confidence interval based on the mean \pm 2.58 S.D. of 3-month young male rats was used to select those aged rats that made the SDL under the lower limit of $99 \%$ confidence interval. 11 aged rats with memory loss were selected, randomly distributed control group $(\mathrm{n}=5)$ and catalpoltreated group $(n=6)[6,7]$.

\subsection{Drug Treatment}

Catalpol was of analytical grade (purity $>98 \%$ ) and purchased from National Institute for the Control of Pharmaceutical and Biological products (China) and dissolved in physiological saline. The catalpol-treated animals received daily once intraperitoneal injection of catalpol $(5 \mathrm{mg} / \mathrm{kg}$ dissolved in a total volume of $1 \mathrm{ml}$ physiological saline), and rats in control group received $1 \mathrm{ml}$ of physiological saline for 10 days.

\subsection{Behavior Test}

\subsubsection{Open Field Test}

The open-field arena consisted of a $76 \times 76 \mathrm{~cm}$ quadratic, white wooden box, without a cover, divided into 25 equally sized squares and confined by walls of $40-\mathrm{cm}$ high. Testing was conducted between 9 am to 2 pm, under white light in a quiet room. The observation was held for 5 minutes for three time points: before, 5 days and 10 days after the catalpol intraperitoneal injection. At the beginning of the 5-min session, the rat was placed in the center square of the open field and the number of crossing, number of rearing, and time of entering center scored each min for the 5-min. The scores of crossing and rearing regarded as locomotor scores. Individual controlor catalpol-treated animals were placed gently in the center of the arena and were allowed to explore freely for $5 \mathrm{~min}$ before measurement [8].

\subsubsection{Y-Maze Test (Alternative Electro-Stimulus Y-Maze)}

The Y-maze test can be used for investigation of electric foot shock-motivated discrimination learning and memory in rats. The Y-maze consisting of three equal arms $\left(45 \times 14 \times 18 \mathrm{~cm}^{3}\right)$ with a stainless-steel grid floor and black plastic walls was used. A $15 \mathrm{~W}$ incandescent lamp was located on the end of each arm. One arm compartment was lit and designated as safe zone while the other two were unsafe zones. The apparatus placed on the floor 
of experimental room. Each rat was placed in one of the arm compartments and was allowed to move freely for 5 min without reinforcers, and arm entry was defined as the body of a rat except for its tail completely entering into an arm compartment. Then they begin to learn a foot shock-motivated spatial alternation.

At the beginning of the training session, a foot shock (30 V, $0.7-1.5 \mathrm{~mA}$, depending on individual sensitivity) was given in the start arm and the animal had to escape into the bright arm (correct run, no foot shock in this arm), whereas entry into the dark arm (error run) was punished by further foot shock $(30 \mathrm{~V}, 0.7 \mathrm{~mA})$. They soon learn to escape from the dark arms to the bright arm to avoid the pain of foot shocks. In this study, a response was considered to be correct when a rat ran directly to the bright arm within 10 seconds after the onset of foot shocks. Each rat was trained consecutively 20 times. Twenty-four hours after the learning retention (T1), memory retention $(\mathrm{R} \# 1)$ of the $\mathrm{Y}$-maze spatial alternation task was tested using the same behavioral procedure as during training. The learning and memory retention test was run after 5 days (T2, R\#2) and 10 days catalpol treatment (T3, R\#3) again [9].

The following parameters were evaluated: number of learning retention errors, number of memory retention errors and percent correction (number of correct/20 $\times$ 100).

\subsection{Slice Preparation}

Rats were deeply anesthetized by intraperitoneal injection of ketamine and xylazine as determined by the absent of the tail and paw withdrawal reflex 24 hours after last Y-maze test. Slices were prepared as described [10]. Briefly, perfused transcardially with heparinized saline followed by $4 \%$ paraformaldehyde, brain tissue were removed carefully and put quickly into cold physiological saline. The right hemisphere was immediately dissected from the frontal cortex and hippocampus over ice by the Glowinskid method, weighed for chemical analysis. The left hemisphere from aged rats was fixed in $4 \%$ paraformaldehyde for $24 \mathrm{~h}$. Tissues were frozen in serial solutions of $10 \%, 20 \%$, and $30 \%$ sucrose and embedded in paraffin, then cut into $8 \mu \mathrm{m}$ of thickness slices. Thereafter, the slices were immediately transferred to a submerged-type slice chamber and permanently preserved.

All experiments were performed in accordance with the National Institutes of Health guide for the care and use of Laboratory animal (NIH Publications No. 8023) and were approved by the Dalian Medical University, Animals Research Committee. All efforts were made to minimize animal suffering and the number of animals used in this study.

\subsection{HE Staining}

The samples were stained by hematoxylin and eosin $(\mathrm{H}$ $\&$ E). Briefly, the dried tissue sections were stained with $0.1 \%$ Hematoxylin for $5 \mathrm{~min}$, then rinsed in running water for $10 \mathrm{~min}$ and distilled water for a few seconds. After rinse with $95 \%$ ethanol for $5 \mathrm{~s}$, the sections were stained with $0.5 \%$ Eosin for $30 \mathrm{~s}$ and dried in series concentrations of ethanol, mounted in neutral gum and examined under a light microscope.

\subsection{Immunohistochemistry}

Tissue sections were deparaffined and stained with the standard immunohistochemical procedures. Firstly, sections were incubated in $3 \% \mathrm{H}_{2} \mathrm{O}_{2}$ for $30 \mathrm{~min}$ at room temperature to block endogenous peroxidase activity. After washing in PBS for 3 times (2 min/time), sections were preincubated with normal goat serum (added with $5 \% \mathrm{BSA}$ ) for $20 \mathrm{~min}$ at $37^{\circ} \mathrm{C}$, then incubated with primary antibody, rabbit polyclonal antibody-(GAP-43) (1:400, Boster, China) for $1.5 \mathrm{~h}$ at $37^{\circ} \mathrm{C}$, and subsequently incubated with secondary antibody, horseradish peroxidase-labeled goat anti-rabbit(1:2000, Sigma, America) for $30 \mathrm{~min}$ at $37^{\circ} \mathrm{C}$ after washing 3 times $(10 \mathrm{~min} /$ time). Peroxidase activity was detected using 3',3'-diaminobenzidine (DAB) technique. Negative controls were carried out by similarly treating adjacent sections and omitting the primary antibody preabsorbed with antigen excess. Images for analysis were randomly selected. Semiquantitative measurements of IR (immunoreactivity) for GAP-43 from hippocampal CA1 region, CA3 region, dentate gyrus (DG) and frontal cortex regions were quantified using computerized image analysis software (Image J Program, NIH, USA). All sections were coded and data analysis was carried out blindly.

\subsection{Western Blot}

Hippocampus and frontal cortex tissue were prepared on $4{ }^{\circ} \mathrm{C}$ ice-water and added in schizolysis solution $(0.15 \mathrm{M}$ $\mathrm{NaCl}, 1 \%$ Triton $\mathrm{X}-100,1 \%$ deoxycholow, $0.1 \%$ SDS, $10 \mathrm{mM}$ Tris- $\mathrm{HCl} \mathrm{pH} \mathrm{7.4,} \mathrm{1 \%} \mathrm{phenylmethylsulfonylflu-}$ oride), homogenized (10000 r/min, $10 \mathrm{~min}$ ) with icecold saline to split protein. Then the supernatant were frozen at $-20^{\circ} \mathrm{C}$ for western blotting analysis. $100 \mu \mathrm{g}$ of total protein were separated by $12 \%$ sodium dodecyl sulfate-polyacrylamide (SDS) gel electrophoresis for $2 \mathrm{~h}$ and then transferred to PVDF membrane (Hybond, America) (room temperature, $220 \mathrm{~V}$ for $60 \mathrm{~min}$ ). Equal protein loading was verified by Coomassie Blue Staining. Blots were incubated with polyclonal antibody-(GAP-43) (1:400, Boster, China) diluted in $0.1 \%$ phosphate-buffered saline (PBS) containing $0.1 \%$ Tween-20 for overnight at $4{ }^{\circ} \mathrm{C}$. After incubation and 3 times washing in $0.1 \%$ PBS (10 $\mathrm{min} /$ time), the samples were incubated in secondary an- 
tibody, horseradish peroxidase-labeled goat anti-mouse (1:2000, Sigma, America) and rocked for $1 \mathrm{~h}$ at room temperature. After washing 3 times in PBS, membranes were detected via Amersham's Enhanced chemiluminescence kit (Amersham Corp, America)and exposed to Kodak XAR5 films for $30 \mathrm{~s}-3 \mathrm{~min}$. Likewise, the level of $\beta$-actin used as an internal control. Quantitative measurement of protein level was performed using the Image $\mathrm{J}$ (NIH).

\subsection{Statistical Analysis}

Statistical analyses were performed using SPSS 10.0 and for all analysis a probability of less than 0.05 was considered as significant. The mean values for the protein levels were computed for the control and catalpol-treated rats for each group. Student's t-test (two tails, unpaired) was used for two-group comparisons. Linear regression analysis was performed on the individual samples to evaluate association between variables. Graphs were plotted using Excel software. The results were reported as the mean \pm S.E.M. with the S.E.M. represented graphically by error bars.

\section{Results}

\subsection{Effects of Catalpol on Spatial Learning and Memory}

Rats were tested on 3 separate days (before/5 days/10 days after catalpol treatment) in the open field. We aimed to study whether the improvement in learning and memory after catalpol treatment is course-dependent ( 5 days and 10 days), therefore we did this on the same group of animal rats.

After catalpol treatment for 5 days, the number of rearing, crossing and the locomotion scores were markedly increased than aged control group, however, there was no significant difference between catalpol-treated and young control groups on the number of crossing and locomotion scores (Figures 2(a)-(c)). Moreover, the number of rearing of catalpol-treated for 5 days was significantly lower than that of young control groups. While locomotion scores, the number of crossing square, rearing and entering center time all has been significantly improved compared to aged groups for 10 days treatment.

Entries into the center reflect exploratory behavior. The phenotypic difference in center entries time reflected a raise in exploratory behavior in catalpol-treated rats after 10 days treatment (control group: $0.70 \pm 0.23$; catalpol-treated group: $0.26 \pm 0.14, \mathrm{P}<0.05)$. In addition, catalpol-treated animals performed a slightly decrease in center entries time after 5 days treatment, and there was no significant difference in between (control group: 1.01

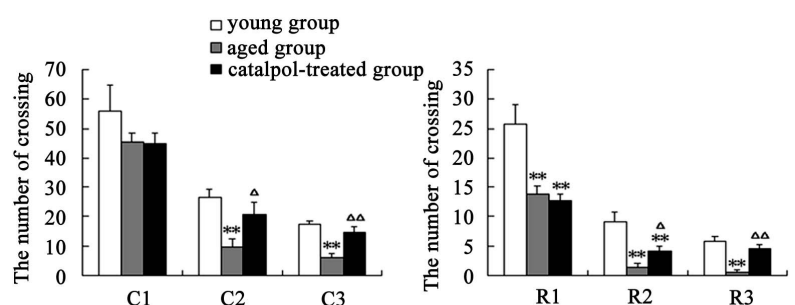

(a)

(b)

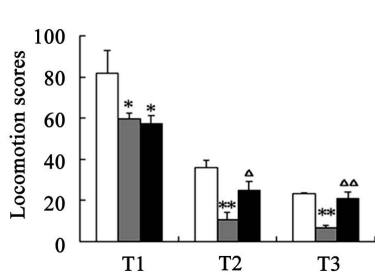

(c)

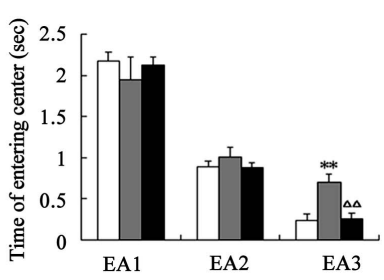

(d)
Figure 2. Effects of catalpol on exploratory activity using open-field test. (a) The number of crossing in horizontal activity; (b) The number of crossing in vertical activity; (c) Locomotion scores; (d) Entering center time. 1, 2, 3 represents time before, 5 days and 10 days after catalpol treatment. Each value represents the mean \pm S.E.M. ( ${ }^{*} \mathrm{P}<0.05$, ${ }^{* *} \mathbf{P}<0.01$ compared to young controls; ${ }^{\triangle} \mathbf{P}<\mathbf{0 . 0 5},{ }^{\triangle \triangle} \mathbf{P}<$ 0.01 compared to aged controls).

\pm 0.27 ; catalpol-treated group: $0.89 \pm 0.16, \mathrm{P}>0.05)$ (Figure 2(d)).

The results of spatial learning and memory in y-maze test were shown in Figure 3. In Y-maze, the number of trial times reached to learning criterion is lower and the rate of correct response to avoid foot shocks is higher in training (T3) and retention (R3) experiment procure for the catalpol-treated rats after 10 days administration compared with aged control group, which indicated catalpol increased spatial learning and memory of aged rats. After 5 days catalpol administration, no significant changes in the number of trial times reached to learning criterion and correct ratio have been observed in catalpol-treated group in training (T2), but correct ratio obviously increased and the number of trial times significantly reduced in retention experiment (R2) in catalpol-treated rats compared with aged control group.

\subsection{Histochemistry Assay}

Microphotographs of neuron structure in rat hippocampus are shown in Figure 4. In both control and catalpol-treated groups, neurons in rat hippocampal CA1 region organized orderly with clear boundaries, the dark stained nucleus were large and clear with well-distributed chromatin and little perinuclear cytoplasm. In hippocampal CA3 region, neurons in both groups were orderly arranged, cell nuclei were round and dark stained. In addition, the astrocytes in catalpol-treated group were more 


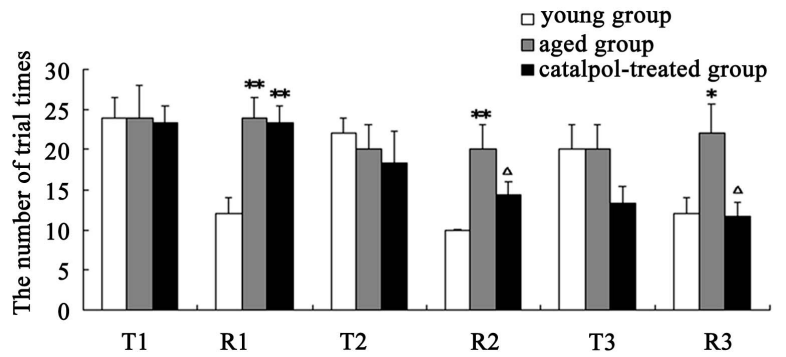

(a)

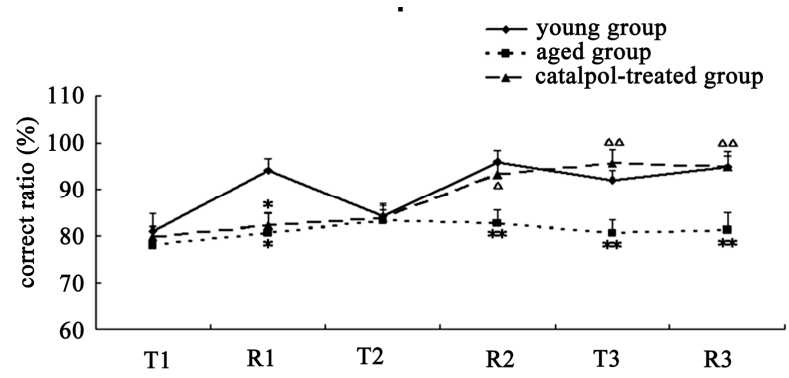

(b)

Figure 3. Effects of catalpol on spatial performance in $\mathrm{Y}$ maze test. (a) The number of trial times reached to criterion; (b) The rate of correct response to avoid foot shocks (correct ratio). $T$, training test; $R$, retention test; $1,2,3$ represents time before, 5 days and 10 days after catalpol treatment. Each value represents the mean \pm S.E.M. ( $P<0.05$, ${ }^{* *} \mathbf{P}<0.01$ compared to young controls; ${ }^{\Delta} \mathbf{P}<0.05,{ }^{\triangle} \Delta \mathbf{P}<$ 0.01 compared to aged controls).

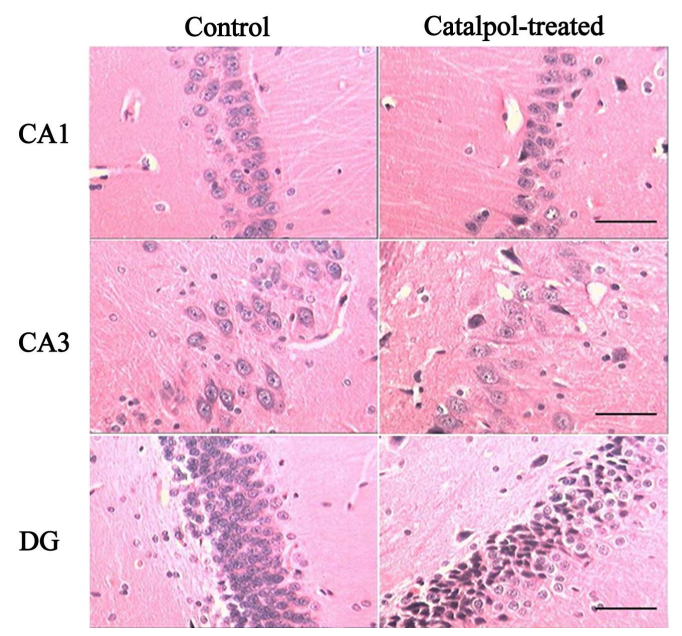

Figure 4. Microphotographs of the neurons in hippocampus tissue by $\mathrm{HE}$ staining $(100 \times)$ before and after catalpol treatment for 10 days. In both control and catalpol-treated groups, neurons in rat hippocampal CA1 and CA3 region organized orderly. In addition, the astrocytes in catalpoltreated group were more than that in control group. Neurons in dentate granule layer of control group were slightly smaller than that of catalpol-treated group. There were fewer neurons but more polygonal astrocytes in dentate granule layer of catalpol-treated group compared to that of control group. DG, dentate granule layer. Scale bars: 200 $\mu \mathrm{m}$. than that in control group. Neurons in dentate granule layer of control group were slightly smaller than that of catalpol-treated group. There were fewer neurons but more polygonal astrocytes in dentate granule layer of catalpol-treated group compared to that of control group.

\subsection{Effects of Catalpol on GAP-43 Protein Levels in Hippocampus and Frontal Cortex}

The distribution of GAP-43 protein containing neurons in the hippocampus was shown in Figures 5 and 6. After catalpol treatment for 10 days, GAP-43 expression in hippocampal CA3 and dentate granule layer was significantly enhanced compared to untreated aged rats. In contrast, levels of GAP-43 protein in the hippocampal CA1 region were not significantly different between catalpol-treated and untreated groups (Figure 5). Since there was no significant difference of GAP-43 expressed in frontal cortex (data not shown), the neurons with GAP43 IR in the hippocampus were further observed and quantified. As shown in Figure 6, quantification of GAP43 protein in hippocampal CA3 region and dentate granule layer revealed remarkable increase in catalpol-treated rats $(\mathrm{P}<0.05)$. There was no difference in levels of GAP43 protein in CA1 region between control group and catalpol-treated group $(\mathrm{P}>0.05)$.

Western blot analysis demonstrated a catalpol-associated increase of GAP-43 in hippocampus after 10 days treatment than that of control group $(\mathrm{P}<0.01)$ (Figures 7(a) and (c)). However, there was no significant difference in GAP-43 protein expression frotal cortex between the untreated and catalpol-treated groups (Figures 7(b) and (d)).

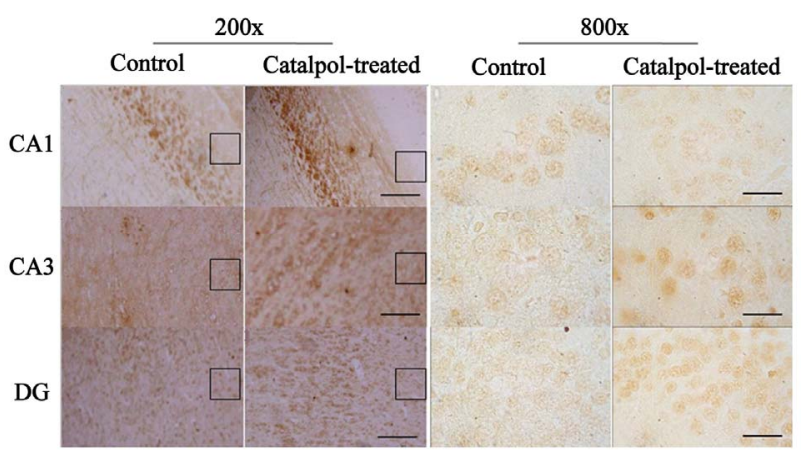

Figure 5. Microphotographs showing GAP-43 immunostaining (left) and neurons with GAP-43-IR (right) in the hippocampal regions of rats before and after catalpol treatment for 10 days. Immunohistochemistry staining showed the increase of GAP-43 protein in the hippocampal CA3 region and DG of catalpol-treated rats compared to that of aged rats (control group), but no obvious changes were found in hippocampal CA1 region. The smaller boxed areas were corresponded to the regions of the hippocampal regions in high magnification. DG, dentate granule layer. Scale bars: $25 \mu \mathrm{m}$ (left column), $100 \mu \mathrm{m}$ (right column). 


\subsection{Correlation between GAP-43 Protein Expression and Spatial Task}

The hippocampal GAP-43 protein level was correlated with scores of locomotion $(\mathrm{r}=0.850, \mathrm{P}=0.032)$ (Figure 8(a) $)$ and entering center time $(\mathrm{r}=-0.936, \mathrm{P}=0.006)$ (Figure 8(b)) in open-field test. Increasing in GAP-43 protein in hippocampus was also correlated with the reduction of percent errors in memory retention (R\#3) in Y-maze test after 10 days catalpol treatment $(\mathrm{r}=0.821, \mathrm{P}$ $=0.045$ ) (Figure 8(d)), but not with that in learning retention $(\mathrm{T} 3)(\mathrm{r}=0.596, \mathrm{P}=0.212)($ Figure 8(c)).

\section{Discussion}

Animal models have played a major role in studying the behavioral and neuronal mechanisms underlying drug effects [11]. In the present study, we aimed to determine whether catalpol can regulate central neuroplasticity in aged rats with memory loss. Aging has negative effects on neuroplasticity and consequently, on learning and memory. For example, a study has showed that there is reduction of GAP-43 levels in hippocampus of aged rats [12]. The decreased capacity for plasticity with age might represent a continuous process of which aged-related memory loss is an inevitable endpoint. Nowadays there is consensus that age-related changes in the neuroplasticity may play an important role on the on-set and progression of aged-related memory loss [13]. Based on that, we used spontaneous aged rats, which could fully exhibit the age-related typical neuroplasticity changes and impairment in tasks of spatial learning, attention and memory [11].

\subsection{Catalpol Increases Spatial Performance and Behavior Response}

Previous studies have shown that catalpol treatment of

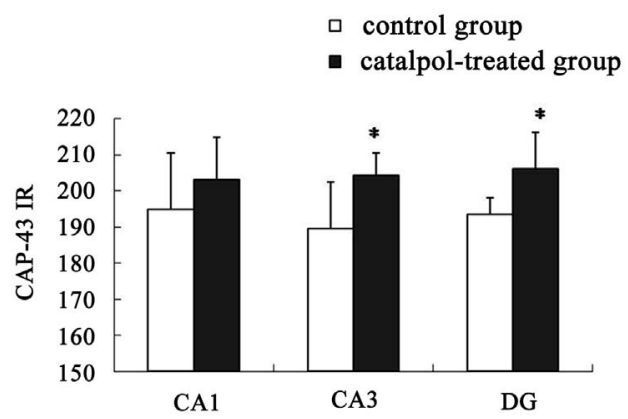

Figure 6. Semi-quantitative measurement of GAP-43 immunoreactivity (GAP-43 IR) was quantified in hippocampal regions. No significant changes in GAP-43 protein expression were observed in hippocampal CA1 region between catalpol-treated rats and untreated aged rats. Each value represents the mean \pm S.E.M. ( ${ }^{*} P<0.05$ compared to controls). DG, dentate granule layer. ischemic animal models can increase cognition on the Y-maze [4]. In the present study, we confirmed that catalpol can reverse spatial learning and memory deficits and demonstrated the effects of catalpol on behavioral dysfunction of aged rats with memory loss.

We used Y-maze to investigate the role of catalpol on special memory in aged rats. Y-maze is widely used for identification of discrimination learning, spatial alteration tasks, working and reference memory, particularly as an evaluating test of memory function in $\mathrm{AD}$ [9]. Numerous studies have reported the aged rats and transgenic rats of $\mathrm{AD}$ models displayed a severe deterioration in the spatial learning and memory on the Y-maze $[14,15]$. Our

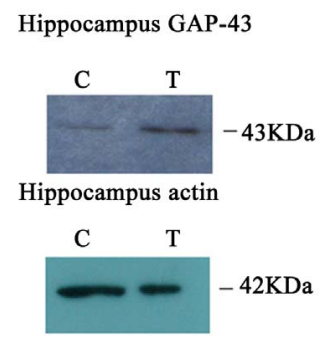

Hippocampus GAP-43

(a)
Frontal cortex GAP-43

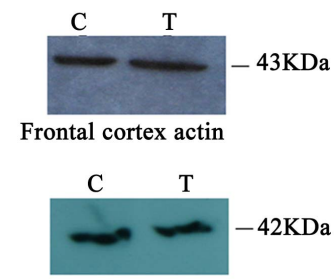

Frontal cortex GAP-43

(b)

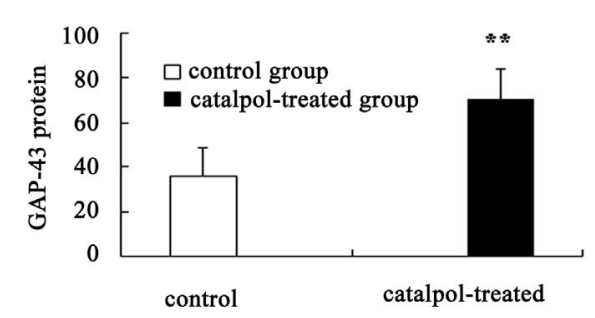

(c)

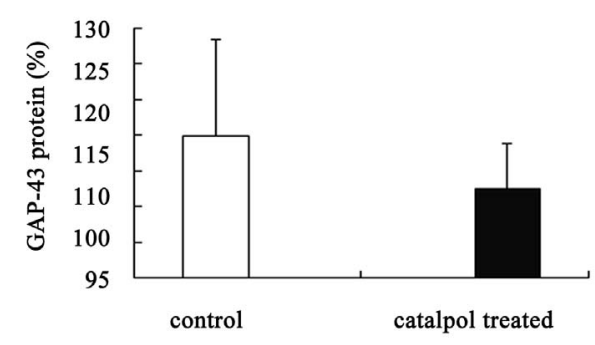

(d)

Figure 7. Effects of catalpol on GAP-43 expression in hippocampus and frontal cortex. Western blots from catalpol-treated rats demonstrated improved GAP-43 expression in hippocampus (a), but not in frontal cortex (b) compared with untreated aged rats. Densitometric analysis of blots showed that the total amount of GAP-43 protein in hippocampus of catalpol-treated rats was significantly more than that of untreated aged rats (c). However, there was no change in GAP-43 protein expression in frontal cortex between two groups (d). C, control group; T, catalpol-treated group. 


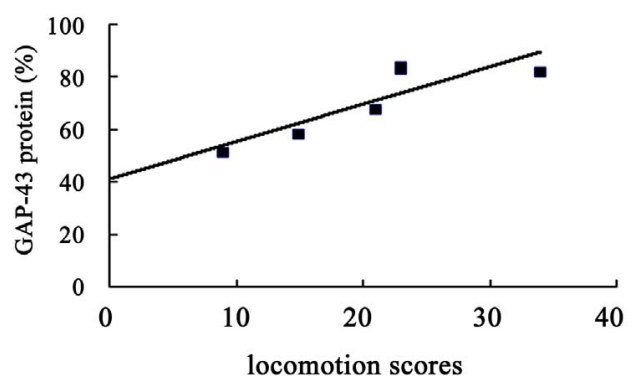

(a)

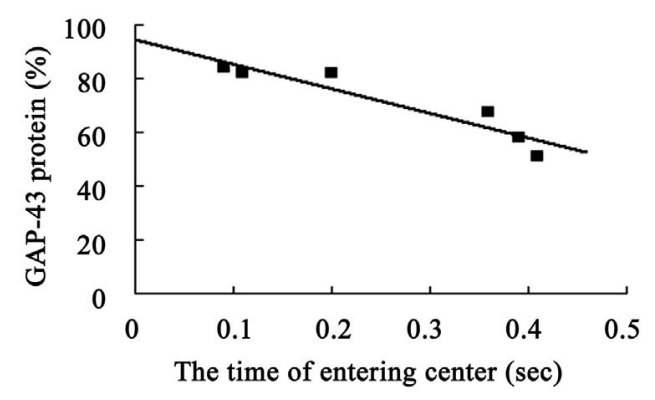

(b)

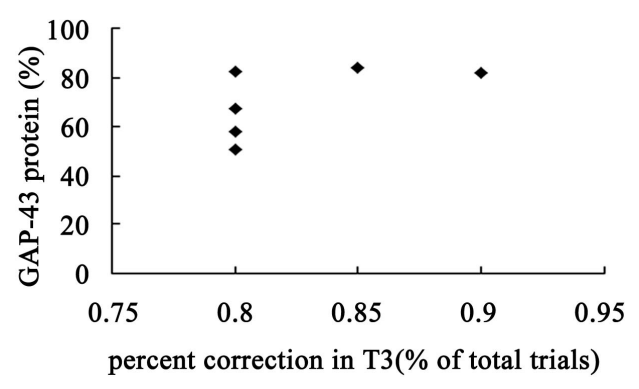

(c)

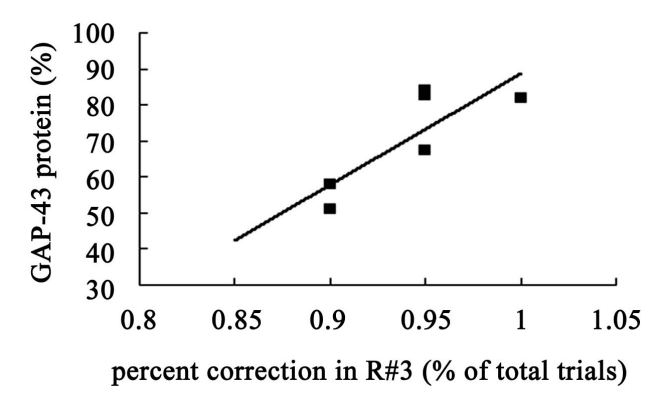

(d)

Figure 8. Correlation between hippocampal GAP-43 protein expression and spatial tasks. The results represented a positive correlation between hippocampal GAP-43 protein and the locomotion scores in open field test $(\mathrm{a}, \mathrm{r}=\mathbf{0 . 8 5 0}, \mathrm{P}=$ 0.032), while the entering center time were in reverse ratio $(b, r=-0.936, P=0.006$ ). The levels of $G A P-43$ were positively correlated with the percent correction in memory retention $(\mathrm{R} \# 3)$ within the $\mathrm{Y}$-maze test $(\mathrm{d}, \mathrm{r}=\mathbf{0 . 8 2 1}, \mathrm{P}=$ $0.045)$ and there was no correlation between GAP- 43 protein and percent correction in learning retention (T3) $(c, r=$ 0.596, $P=0.212$ ) after 10 days catalpol treatment. Each value represents the mean \pm S.E.M $\left(^{* *} P<0.01\right.$ compared to controls). results showed that catalpol could decrease the number of trials to reach learning criterion and reduce the times of mistake, as well as improve the correct ratio of escaping foot shocks in Y-maze task during training and retention experiment procedure after 10 days treatment, which indicated catalpol can ameliorate the impairment of spatial learning and memory of aged rat. Cui et al. has reported rehmannia could improve the function of learning and memory of $\mathrm{AD}$ rats, like decreasing the times of mistakes and prolonging the incubation period in step down task, shortening the incubation period of seeking the platform, and improving the rate through the platform position in Morris water maze task [16]. Similarly, the learning and memory of aged rats treated with rehmannia compound also have been improved [17]. Wang et al. pointed out that catalpol could improve memory and protects the forebrain neurons from neurodegeneration through increasing brain-derived neurotrophic factor (BDNF) expression [18]. Although there are few studies on the effect of catalpol on memory deficits of aged rats with memory loss, our previous studies have found an increase in spatial memory in ischemic animal models that have received catalpol treatment $[4,19]$. Together with our present experimental results, it seemed that catalpol may play an important role on memory deficits induced by injury and aging and it may reverse age-associated spatial memory abnormalities of aged models.

The progressive impairment of cognitive functions in aged often parallels with other abnormal behavior functions such as exploratory, attention and emotion [11]. In this study, we used open-field test to investigate the behaviors in aged rats. The open-field test is the most widely test in animal psychology, which is used to measure locomotor activity and exploratory [8]. Some studies demonstrated that both locomotor activity and exploratory, which reflected responses to novelty, were reduced in aged animal models with memory loss [20].

The evaluation of the different behavioral measurements obtained in the open-field test provided clear evidence that application of catalpol would induce obvious behavioral alteration. Catalpol-treated animals performed high scores of locomotor and shorter entering center time, which were involved in the magnitude of activity and exploratory motivation, respectively. These new finding demonstrated that catalpol could prevent behavioral deficits in an aged model.

\subsection{Catalpol Enhances GAP-43 Level in the Hippocampus}

Although there are several reports about an involvement of catalpol in learning and memory impairment, the mechanisms that regulate cognition still remain unknown. One possible mechanism may be the neuroplasticity re- 
gulation. To determine whether catalpol could influ- ence neuroplasticity, we examined the GAP-43 level in hippocampus and frontal cortex of aged rats that received catalpol administration. GAP-43 is a growth-associated phosphoprotein expressed at high levels in neurons during development, axonal regeneration, and neuritic sprouting, which is probably functionally important for the structural remodeling of synapses as required for learning and establishing new memory. It is involved in maturational and plasticity-associated processes, and changes in GAP-43 expression are a marker of altered plasticity following experimental and neuropathological lesions [21].

In immunohistochemistry, we found that there was a clear raise of GAP-43 level in the hippocampus after catalpol treatment. Semi-quantitative analysis showed that GAP-43 IR in hippocampal CA3 region and dentate gyrus in the hippocampus had been markedly increased. The results of western blotting were consistent with the immunohistochemical results, i.e., increased levels of GAP-43 in hippocampus. Previous analysis has shown that GAP-43 is significantly lower in aged rats than controls in the hippocampus [21]. The finding is supported by the majority of studies investigating the levels of GAP-43 in various brain regions of AD patients [22,23]. We once hypothesized that catapol could increase presynaptic proteins and up-regulate relative signaling molecules in the hippocampus of the aged rats [10]. Downregulated and aberrant neuronal GAP-43 expression appears to indicate an important molecular lesion that precedes and progresses with the widespread synaptic disconnection and dementia in AD [24]. Our results showed that the decreased GAP-43 level in aged rats can be ameliorated, at least in part of, by catalpol, which suggests that catalpol may overcome some age-related deficits in neuroplasticity.

Previously experiments showed that GAP-43 was also significantly lower in aged rats in the frontal cortex [21]. However, we did not find catalpol increase GAP-43 protein levels in frontal cortex, which suggested that catalpol may mainly affect hippocampal neuroplasticity. One explanation of why neurons in hippocampus and frontal cortex respond to catalpol differently may be the selective neuronal vulnerability to aging. Brain regions, particularly those involved in learning and memory, which demonstrate neurons within the entorhinal cortex (EC) and hippocampus degenerate in AD are particularly vulnerable to the consequences of chronic neuroinflammation and aging [25]. Evidence for loss of hippocampal neuroplsticity is common in the brain of aged dementia patients [22]. These results indicate that intrinsic differences of brain regions in the neuronal vulnerability linked to neuroplasticity may account for selective action of catalpol. Neurons with a high degree of vulnerability to aging are apt to respond to catalpol.
Indeed, even within highly vulnerable regions, such as hippocampus, neurons are affected to a variable extent, which may explain why catalpol selectively increases GAP-43 expression in CA3 and DG regions. It has been documented that selective susceptibility affects hippocampal regions to a different extent. CA3 pyramidal cells were mostly affected in normal aging and following hypobaric hypoxia, whereas CA1 cells were especially affected following corticosterone administration, global ischemia and ChE inhibition [26]. The hippocampal CA3 and DG neurons are particularly vulnerable to degeneration and death in association with aging, whereas CA1 neurons show little evidence of degeneration during normal aging or in aged dementia [27]. One study has showed that the baseline levels of GAP-43 mRNA decreased with age, especially in the CA3 region [12]. Therefore, these results indicated that neuroplasticity of some regions in the brain vulnerable to age-related degeneration is more likely to recover after catalpol treatment.

\subsection{Increased Hippocampal Levels of GAP-43 Are Correlated with Spatial Memory and Behavior Response}

The present results also suggested that little difference in hippocampal GAP-43 expression could be critical for spatial performance and behavior response in aged rats with memory loss. Correlation analysis showed that the increased hippocampal levels of GAP-43 induced by catalpol treatment was associated with spatial memory performance, those rats with higher hippocampal GAP43 expression required less trial times to reach learning criterion, and they showed more correct response to avoid foot shocks in the retention experiment. Studies by others have suggested the amount of GAP-43 in the hippocampus is significantly correlated with performance in the memory task $[3,28]$, and they are consistent with our findings. We can draw a conclusion that catalpol may increase memory by up-regulating GAP-43 level in the hippocampus of aged dementia rats. Data from our studies provided an indirect evidence to support the hypothesis that the level of GAP-43 would determine the achievement level of memory-associated performance.

Results also showed that the increased hippocampal GAP-43 is positively correlated with locomotor scores and reversely associated with the time entering center. Animals with higher GAP-43 levels performed better locomotor activity and exploratory behavior in open field test. Our data provided the first evidence of a possible role for catalpol in the rescuing behavioral deficits in aged animals by affecting hippocampal GAP-43 level. Since there are few studies about the involvement of GAP-43 level and behavioral response, further studies 
are required to clarity the interaction between behavioral function, GAP-43 expression and catalpol. The current data at least supported a new concept that catalpol can increase GAP-43 levels of hippocampus, consequently block loss of neuroplasticity and rescue behavior deficits in aged rats.

Thus, it is speculated that catalpol may enhance spatial memory and behavior response of aged rats via upregulating GAP-43 level in hippocampus, particular in some regions such as CA3 and DG. The increments in GAP-43 expression will act to enhance the network plasticity potential on affected regions. One potential mechanism of catalpol leading to the observed increase of hippocampal neuroplasticity can be that catalpol can boost deafferentation resulting from plasticity. Indeed, just such a response of deafferentation has been observed in PC12 cells of catalpol-treated [29].

Certainly, our new findings only initially suggested a positive role of catalpol on age-related loss of neuroplsticity as many other factors may participate in neuroplasticity loss in aged-related memory loss process. In addition, Y-maze test only evaluate some aspects of cognitive function. Further studies are needed to examine the effect of catalpol on cognitive deficits and neuroplasticity loss using AD models and stronger learning tests.

In conclusion, the present results provided evidence to support memory enhancing effects of catalpol and suggested catalpol may have a potential effect on improving behavior response of aged-related memory loss. The more important contribution of the present study is to demonstrate that the protecting effect of catalpol on neuron is, in part of, due to neuroplasticity alterations via up-regulating GAP-43 level in hippocampus. The results suggested catalpol may be a useful natural drug for memory loss with aging treatment by modulating hippocampal neuroplasticity.

\section{Acknowledgements}

This work was supported by grants from the National Natural Science Foundation of China (No. 81071009), Science and Technology Department of Liaoning Province (No. 20091020) and sponsored by the Scientific Research Foundation for the Returned Overseas Chinese Scholars, State Education Ministry.

\section{REFERENCES}

[1] W. C. Shyu, K. W. Li, H. F. Peng, et al., "Induction of GAP-43 Modulates Neuroplasticity in PBSC (CD34 $\left.{ }^{+}\right)$ Implanted-Parkinson's Model," Journal of Neuroscience Research, Vol. 87, No. 9, 2009, pp. 2020-2033. doi:10.1002/jnr.22027

[2] J. Guevara, H. Dilhuydy, B. Espinosa, et al., "Coexistence of Reactive Plasticity and Neurodegeneration in Alzhei- mer Diseased Brains," Histology and Histopathology, Vol. 19, No. 4, 2004, pp. 1075-1084.

[3] J. L. Rekart, K. Meiri and A. Routtenberg, "Hippocampal-Dependent Memory Is Impaired in Heterozygous GAP-43 Knockout Mice," Hippocampus, Vol. 15, No. 1, 2005, pp. 1-7. doi:10.1002/hipo.20045

[4] D. Q. Li, Y. L. Duan, Y. M. Bao, C. P. Liu, Y. Liu and L. J. An, "Neuroprotection of Catalpol in Transient Global Ischemia in Gerbils," Neuroscience Research, Vol. 50, No. 2, 2004, pp. 169-177. doi:10.1016/j.neures.2004.06.009

[5] B. Jiang, J. H. Liu, Y. M. Bao and L. J. An, "Catalpol Inhibits Apoptosis in Hydrogen Peroxide-induced PC12 Cells by Preventing Cytochrome c Release and Inactivating of Caspase Cascade," Toxicon, Vol. 43, No. 1, 2004, pp. 53-59. doi:10.1016/j.toxicon.2003.10.017

[6] F. H. Gage, A. Bjorklund, V. Stenevi, S. B. Dunnett and P. A. Kelly, "Intrahippocampal Septal Grafts Ameliorate Learning Impairments in Aged Rats," Science, Vol. 225, No. 4661, 1984, pp. 533-536. doi:10.1126/science.6539949

[7] K. Sidik and R. W. Morris, "Nonparametric Step-Down Test Procedures for Finding Minimum Effective Dose," Journal of Biopharmaceutical Statistics, Vol. 9, No. 2, 1999, pp. 217-240. doi:10.1081/BIP-100101173

[8] S. M. Brudzynski and S. Krol, "Analysis of Locomotor Activity in the Rat: Parallelism Index, a New Measure of Locomotor Exploratory Pattern," Physiology \& Behavior, Vol. 62, No. 3, 1997, pp. 635-642. doi:10.1016/S0031-9384(97)00189-3

[9] A. Routtenberg, "Measuring Memory in a Mouse Model of Alzheimer's Disease," Science, Vol. 277, No. 5327, 1997, pp. 839-840. doi:10.1126/science.277.5327.839

[10] J. Liu, Q. J. He, W. Zou, et al., "Catalpol Increases Hippocampal Neuroplasticity and Up-Regulates PKC and BDNF in the Aged Rats," Brain Research, Vol. 1123, No. 1, 2006, pp. 68-79. doi:10.1016/j.brainres.2006.09.058

[11] A. A. Oliveira Jr and H. M. Hodges, "Alzheimer's Disease and Neural Transplantation as Prospective Cell Therapy," Current Alzheimer Research, Vol. 2, No. 1, 2005, pp. 7995. doi:10.2174/1567205052772759

[12] H. Schmoll, S. Ramboiu, D. Platt, J. G. Herndon, C. Kessler and A. Popa-Wagner, "Age Influences the Expression of GAP-43 in the Rat Hippocampus Following Seizure," Gerontology, Vol. 51, No. 4, 2005, pp. 215-224. doi:10.1159/000085117

[13] B. Teter and J. W. Ashford, "Neuroplasticity in Alzheimer's Disease," Journal of Neuroscience Research, Vol. 70, No. 3, 2002, pp. 402-437. doi:10.1002/jnr.10441

[14] G. W. Arendash, M. N. Gordon, M. Diamond, et al., "Behavioral Assessment of Alzheimer's Transgenic Mice Followinglong-Term A $\beta$ Vaccination: Task Specificity and Correlations between A $\beta$ Deposition and Spatial Memory," DNA and Cell Biology, Vol. 20, No. 11, 2001, pp. 737-744. doi:10.1089/10445490152717604

[15] H. Takatsu, K. Owada, K. Abe, M. Nakano and S. Urano, "Effect of Vitamin E on Learning and Memory Deficit in Aged Rats," Journal of Nutritional Science and Vitamino- 
logy, Vol. 55, No. 5, 2009, pp. 389-393. doi: $10.3177 /$ jnsv. 55.389

[16] Y. Cui, Z. Yan, S. Hou and Z. Chang, "Effect of Radix Rehmanniae Preparata on the Expression of c-Fos and NGF in Hippocampi and Learning and Memory in Rats with Damaged Thalamic Arcuate Nucleus," Zhong Yao Cai, Vol. 27, No. 8, 2004, pp. 589-592.

[17] J. C. Hou, C. X. Zhang and S. L. Yang, "Effects of Dihuang Compound on Learning and Memory of Rats and Apoptosis of Hippocampal Cells in Alzheimer's Disease," Progress of Anatomical Sciences, Vol. 11, No. 2, 2005, pp. 111-113.

[18] Z. Wang, Q. Liu, R. Zhang, S. Liu, Z. Xia and Y. Hu, "Catalpol Ameliorates Beta Amyloid-induced Degeneraion of Cholinergic Neurons by Elevating Brain-Derived Neurotrophic Factors," Neuroscience, Vol. 163, No. 4, 2009, pp. 1363-1372. doi:10.1016/j.neuroscience.2009.07.041

[19] D. Q. Li, Y. M. Bao, J. J. Zhao, C. P. Liu, Y. Liu and L. J. An, "Neuroprotective Properties of Catalpol in Transient Global Cerebral Ischemia in Gerbils: Dose-Response, Therapeutic Time-Window and Long-Term Efficacy," Brain Research, Vol. 1029, No. 2, 2004, pp. 179-185. doi:10.1016/j.brainres.2004.09.041

[20] N. Traissard, K. Herbeaux, B. Cosquer, H. Jeltsch, B. Ferry, R. Galani, A. Pernon, M. Majchrzak and J. C. Cassel, "Combined Damage to Entorhinal Cortex and Cholinergic Basal Forebrain Neurons, Two Early Neurodegenerative Features Accompanying Alzheimer's Disease: Effects on Locomotor Activity and Memory Functions in Rats," Neuropsychopharmacology, Vol. 32, No. 4, 2007, pp. 851-871. doi:10.1038/sj.npp.1301116

[21] N. Bogdanovic, P. Davidsson, I. Volkmann, B. Winblad and K. Blennow, "Growth-Associated Protein GAP-43 in the Frontal Cortex and in the Hippocampus in Alzheimer's Disease: An Immunohistochemical and Quantitative Study," Journal of Neural Transmission, Vol. 107, No. 4, 2000, pp. 463-478. doi:10.1007/s007020070088
[22] J. L. Rekart, M. M. Quinn, M. Mesulam and A. Routtenberg, "Subfield-Specific Increase in Brain Growth Protein in Postmortem Hippocampus of Alzheimer's Patients," Neuroscience, Vol. 126, No. 3, 2004, pp. 579-584. doi:10.1016/j.neuroscience.2004.03.060

[23] M. Sjogren, L. Minthon, P. Davidsson, et al., "CSF Levels of Tau, Beta-amyloid(1-42) and GAP-43 in Frontotemporal Dementia, Other Types of Dementia and Normal Aging," Journal of Neural Transmission, Vol. 107, No. 5, 2000, pp. 563-579. doi:10.1007/s007020070079

[24] S. M. de la Monte, S. C. Ng and D. W. Hsu, "Aberrant GAP-43 Gene Expression in Alzheimer's Disease," American Journal of Pathology, Vol. 147, No. 4, 1995, pp. 934946.

[25] L. De Toledo-Morrell, M. P. Sullivan, F. Morrell, R. S. Wilson, D. A. Bennett and S. Spencer, “Alzheimer's Disease: In Vivo Detection of Differential Vulnerability of Brain Regions," Neurobiology of Aging, Vol. 18, No. 5, 1997 , pp. 463-468. doi:10.1016/S0197-4580(97)00114-0

[26] T. Kadar, S. Dachir, B. Shukitt-Hale and A. Levy, "Subregional Hippocampal Vulnerability in Various Animal Models Leading to Cognitive Dysfunction," Journal of Neural Transmission, Vol. 105, No. 8-9, 1998, pp. 9871004. doi: $10.1007 / \mathrm{s} 007020050107$

[27] J. H. Morrison, "Hof PR: Selective Vulnerability of Corticocortical and Hippocampal Circuits in Aging and Alzheimer's Disease," Progress in Brain Research, Vol. 136, 2002, pp. 467-486. doi:10.1016/S0079-6123(02)36039-4

[28] M. R. Holahan, K. S. Honegger, N. Tabatadze and A. Routtenberg, "GAP-43 Dene Expression Regulates Information Storage," Learning \& Memory, Vol. 14, No. 6, 2007, pp. 407-415. doi:10.1101/lm.581907

[29] M. Yamazaki, K. Chiba and T. Mohri, "Neuritogenic Effect of Natural Iridoid Compounds on PC12 Cells and Its Possible Relation to Signaling Protein Kinases," Biological \& Pharmaceutical Bulletin, Vol. 19, No. 6, 1996, pp. 791-795. doi:10.1248/bpb.19.791 